Supporting Information for "Can We Reasonably Predict Chronic Species Sensitivity Distributions from Acute Species Sensitivity Distributions?"

Authors: Kyoshiro Hiki ${ }^{\mathrm{a}}$, and Yuichi Iwasaki ${ }^{\mathrm{b}}{ }^{*}$

${ }^{a}$ Center for Health and Environmental Risk Research, National Institute for Environmental Studies, Tsukuba, Ibaraki, 305-8506, Japan

${ }^{\mathrm{b}}$ Research Institute of Science for Safety and Sustainability, National Institute of Advanced Industrial Science and Technology, Tsukuba, Ibaraki, 305-8569, Japan

*Address correspondence to yuichiwsk@gmail.com

\title{
List of materials
}

Table S1

Table S2

Figure $\mathrm{S} 1$

Figure S2

Figure S3

Figure S4

Figure S5

Figure S6

Figure S7

Figure S8

An example of $\mathrm{R}$ code 
Table S1. Median proportions (\%) of algal, fish, and invertebrate species in acute and chronic species sensitivity distributions (SSDs) for chemicals with three different modes of action.

\begin{tabular}{lllll}
\hline & & Narcotic & Specifically & Unclassified \\
\hline Acute & Algae & $22(0-75)$ & $5(0-85)$ & $15(0-71)$ \\
& Fish & $45(0-83)$ & $46(8-65)$ & $40(0-94)$ \\
& Invertebrate & $33(4-68)$ & $42(8-63)$ & $33(6-65)$ \\
& Algae & $40(14-90)$ & $20(0-80)$ & $45(0-89)$ \\
& Fish & $33(0-60)$ & $40(0-67)$ & $27(0-86)$ \\
& Invertebrate & $26(0-57)$ & $38(0-80)$ & $20(0-83)$ \\
\hline
\end{tabular}

The minimum and maximum proportions are shown in parentheses. 
Table S2. Changes in mean and standard deviation (SD) of acute species sensitivity distributions (SSDs) with more than 50 species when removing the most sensitive or tolerant species.

\begin{tabular}{|c|c|c|c|c|c|}
\hline \multirow[b]{2}{*}{ Chemical } & \multirow{2}{*}{$\begin{array}{l}\text { Number } \\
\text { of species }\end{array}$} & \multicolumn{2}{|c|}{$\%$ shift of mean } & \multicolumn{2}{|c|}{$\%$ shift of SD } \\
\hline & & $\begin{array}{r}\text { Most } \\
\text { sensitive }\end{array}$ & $\begin{array}{r}\text { Most } \\
\text { tolerant }\end{array}$ & $\begin{array}{r}\text { Most } \\
\text { sensitive }\end{array}$ & $\begin{array}{r}\text { Most } \\
\text { tolerant }\end{array}$ \\
\hline Cupric oxide & 387 & 0.4 & 0.3 & 2.2 & 1.0 \\
\hline Cadmium chloride & 349 & 0.4 & 0.2 & 1.8 & 0.8 \\
\hline Zinc oxide & 295 & 0.3 & 0.2 & 2.1 & 0.7 \\
\hline Phenol & 217 & 0.3 & 0.1 & 2.6 & 0.7 \\
\hline Malathion & 207 & 0.5 & 0.5 & 1.0 & 0.9 \\
\hline Mercuric nitrate & 191 & 0.7 & 0.6 & 3.0 & 2.0 \\
\hline Lindane & 155 & 0.7 & 0.7 & 1.3 & 1.3 \\
\hline Chlorpyrifos & 153 & 1.7 & 2.1 & 0.9 & 1.4 \\
\hline Lead nitrate & 130 & 0.5 & 0.5 & 1.7 & 1.3 \\
\hline Endosulfan & 126 & 1.7 & 2.3 & 0.8 & 1.7 \\
\hline Copper & 106 & 1.3 & 1.1 & 4.4 & 3.0 \\
\hline Parathion & 103 & 1.8 & 1.5 & 2.1 & 1.2 \\
\hline Nickel chloride & 98 & 0.6 & 0.5 & 2.9 & 1.4 \\
\hline Sodium pentachlorophenate & 89 & 0.9 & 0.8 & 4.4 & 3.0 \\
\hline Permethrin & 84 & 5.0 & 2.5 & 6.6 & 1.1 \\
\hline Dichlorvos & 83 & 1.8 & 1.2 & 3.7 & 1.2 \\
\hline Trichlorfon & 80 & 1.7 & 2.2 & 3.5 & 6.3 \\
\hline Dimethoate & 79 & 1.2 & 0.9 & 2.7 & 1.2 \\
\hline Sodium dodecyl sulfate & 76 & 0.4 & 0.4 & 2.6 & 1.4 \\
\hline Carbofuran & 75 & 1.8 & 1.3 & 5.8 & 2.9 \\
\hline Arsenic(III) sulfide & 72 & 0.8 & 0.4 & 5.2 & 1.2 \\
\hline Silver sulfate & 70 & 1.2 & 2.4 & 1.1 & 6.3 \\
\hline Sodium selenite & 63 & 0.8 & 0.5 & 4.4 & 1.3 \\
\hline 2,4-Dichlorophenoxyacetic acid & 62 & 0.5 & 0.5 & 2.5 & 1.7 \\
\hline Cypermethrin & 62 & 18.8 & 25.8 & 2.3 & 5.1 \\
\hline Azinphos-methyl & 60 & 2.9 & 3.8 & 1.5 & 3.2 \\
\hline Thiobencarb & 57 & 1.0 & 0.8 & 7.3 & 5.1 \\
\hline
\end{tabular}




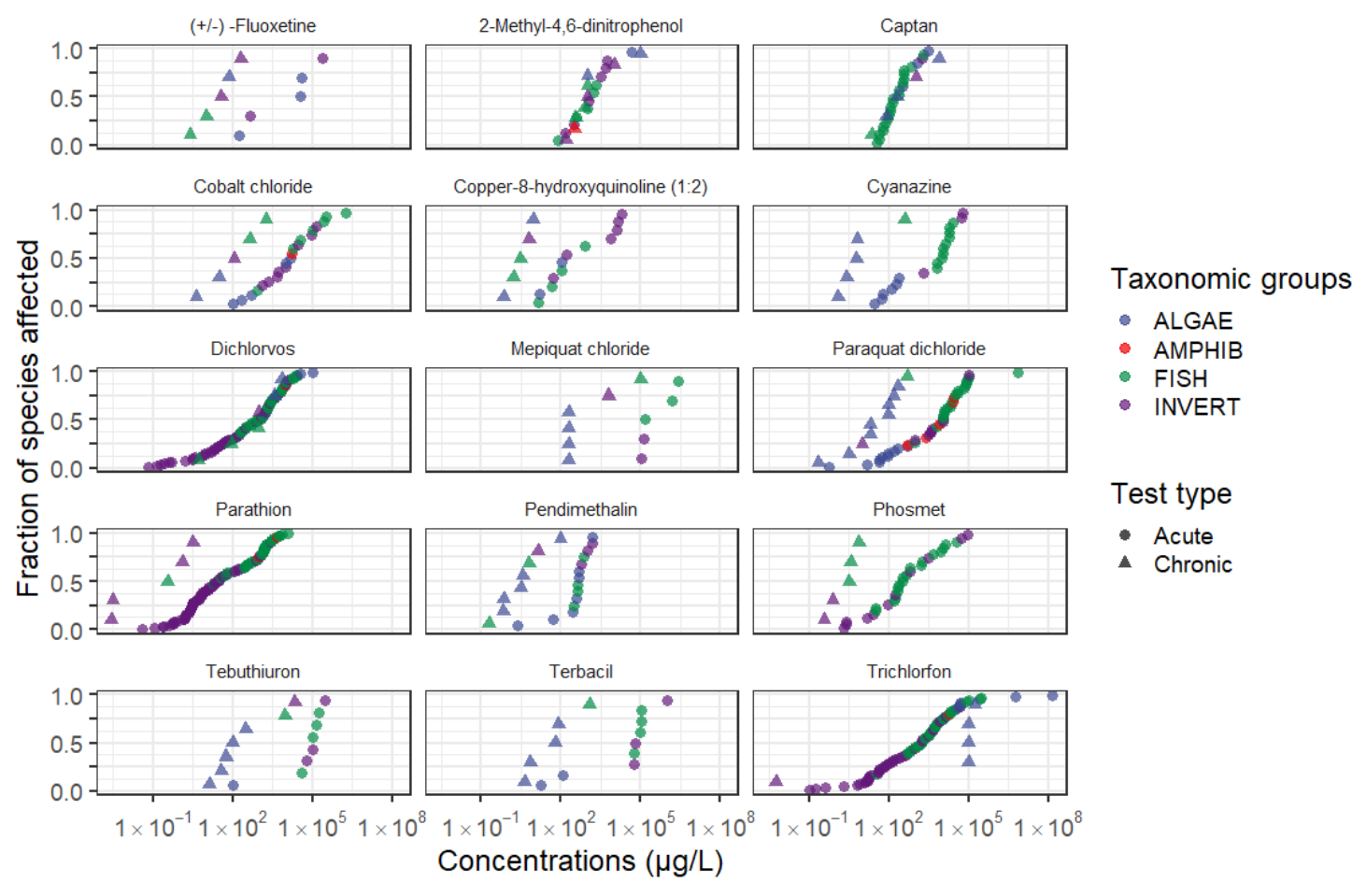

Figure S1. Comparison of acute and chronic species sensitivity distributions (SSDs) for chemicals with the ratios of chronic to acute SSD means outside the range from 1 to 100 (see Figure 2). Circles and triangles represent acute and chronic effect concentrations, respectively. Dot colors represent the different taxonomic groups of the test species. 


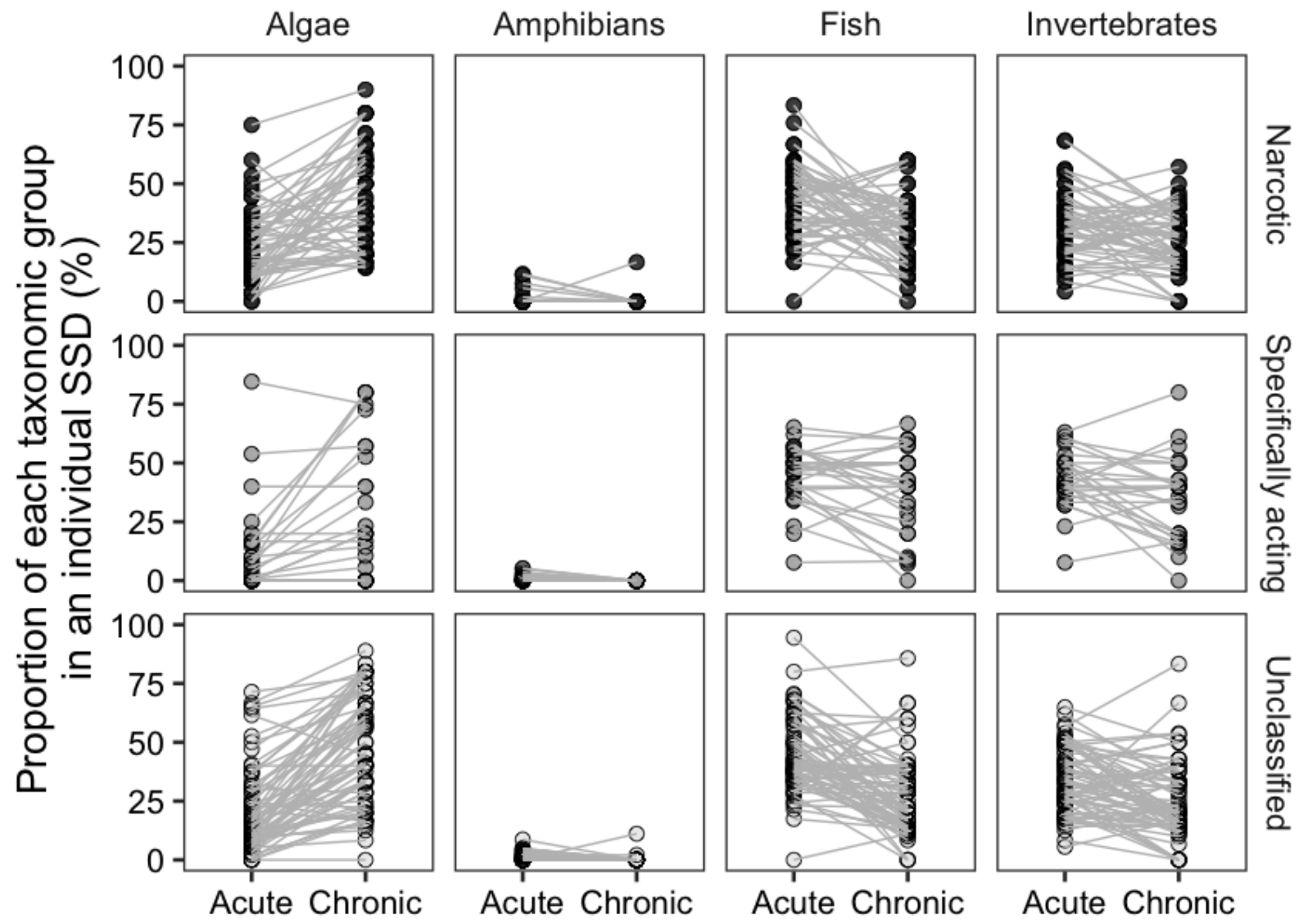

Figure S2. Comparing proportions of taxonomic groups (algae, amphibians, fish, and invertebrates) between acute and chronic SSDs. The same chemicals were connected with grey lines. 


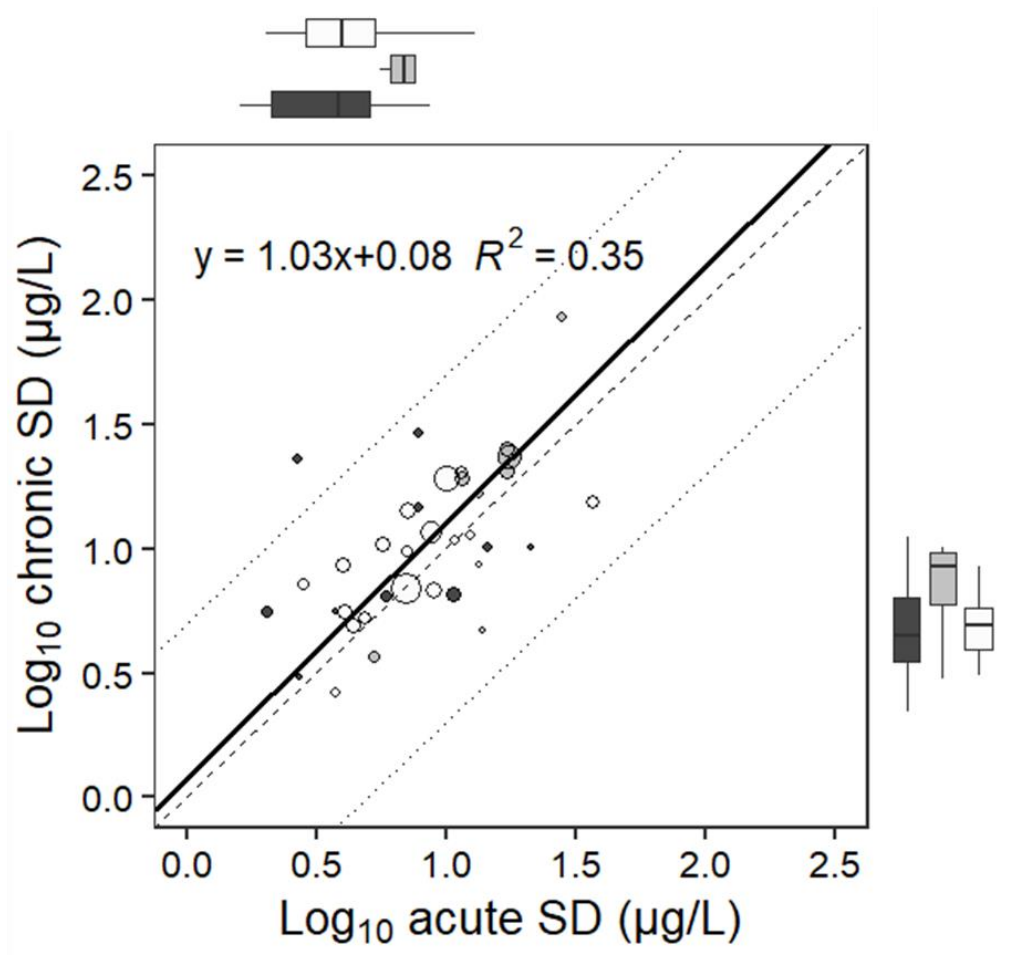

Figure S3. Relationship between the standard deviations (SDs) of acute and chronic species sensitivity distributions (SSDs) when the number of species was $\geq 10$. White, gray, and black dots (and box plots) represent unclassified, specifically acting, and narcotic chemicals, respectively. Further details are as in Figure 1. 


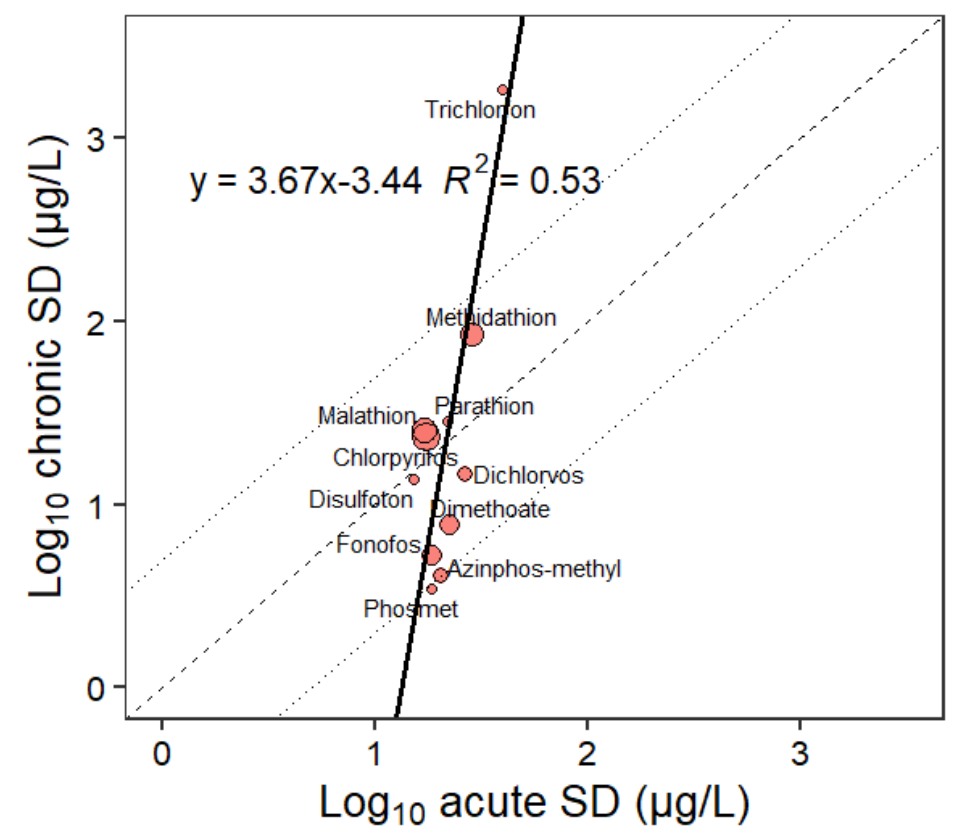

Figure S4. Regression of chronic species sensitivity distribution (SSD) standard deviations (SDs) against acute SSD SDs of only organophosphates that inhibit acetylcholinesterase (MoA assigned by ASTER). The dashed line indicates a 1:1 (acute:chronic) ratio, and the dotted lines indicate ratios of 5:1 and 1:5. The bold line shows the estimated standardized major axis regression. Dot sizes represent the number of test species in the acute or chronic SSDs, whichever is lower. 

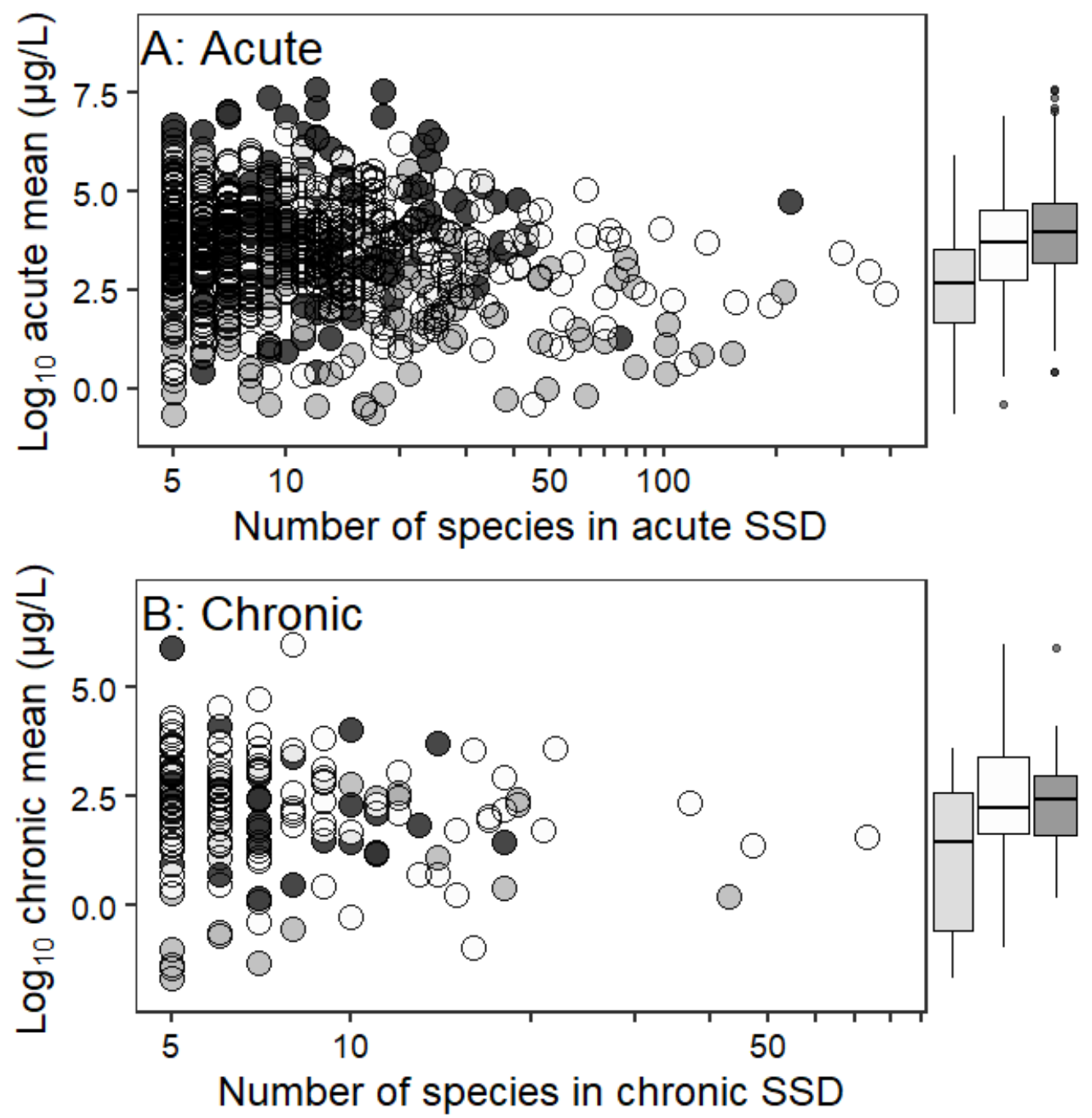

Figure S5. Relationship between the means of (A) 831 acute and (B) 164 chronic species sensitivity distributions (SSDs) and the number of test species in each SSD. White, gray, and black represent unclassified, specifically acting, and narcotic chemicals, respectively. Box plots show the distribution of the means of SSDs for the three modes of action. 


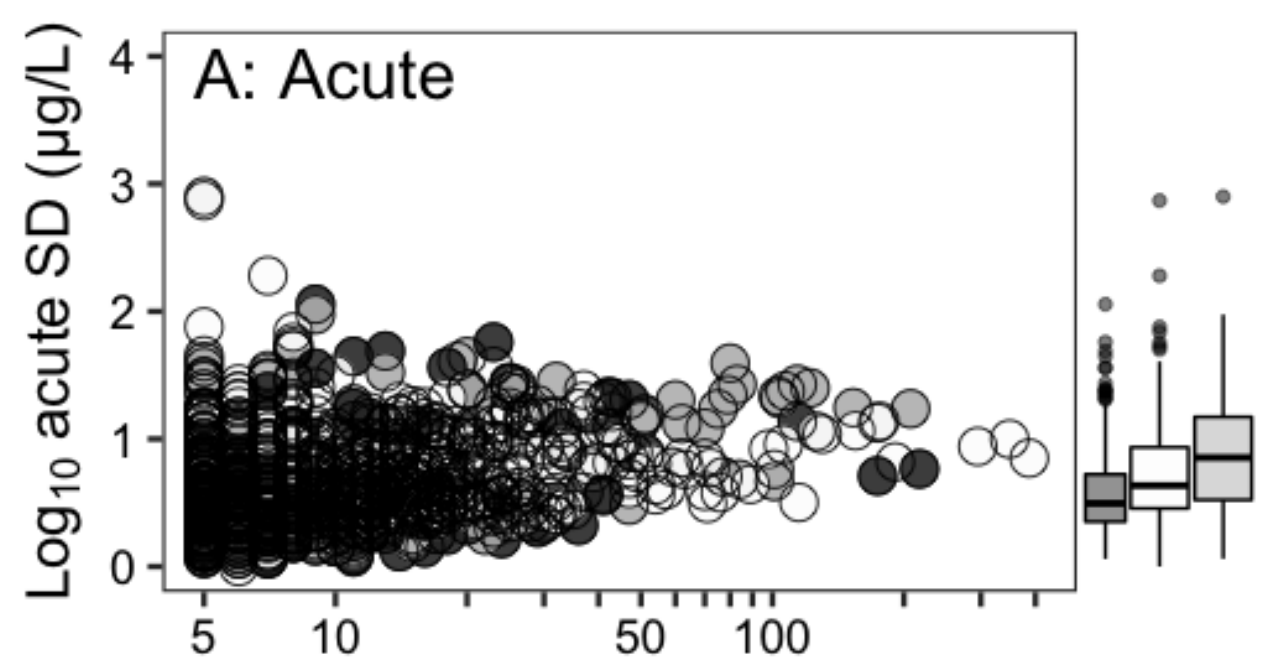

\section{Number of species in acute SSDs}

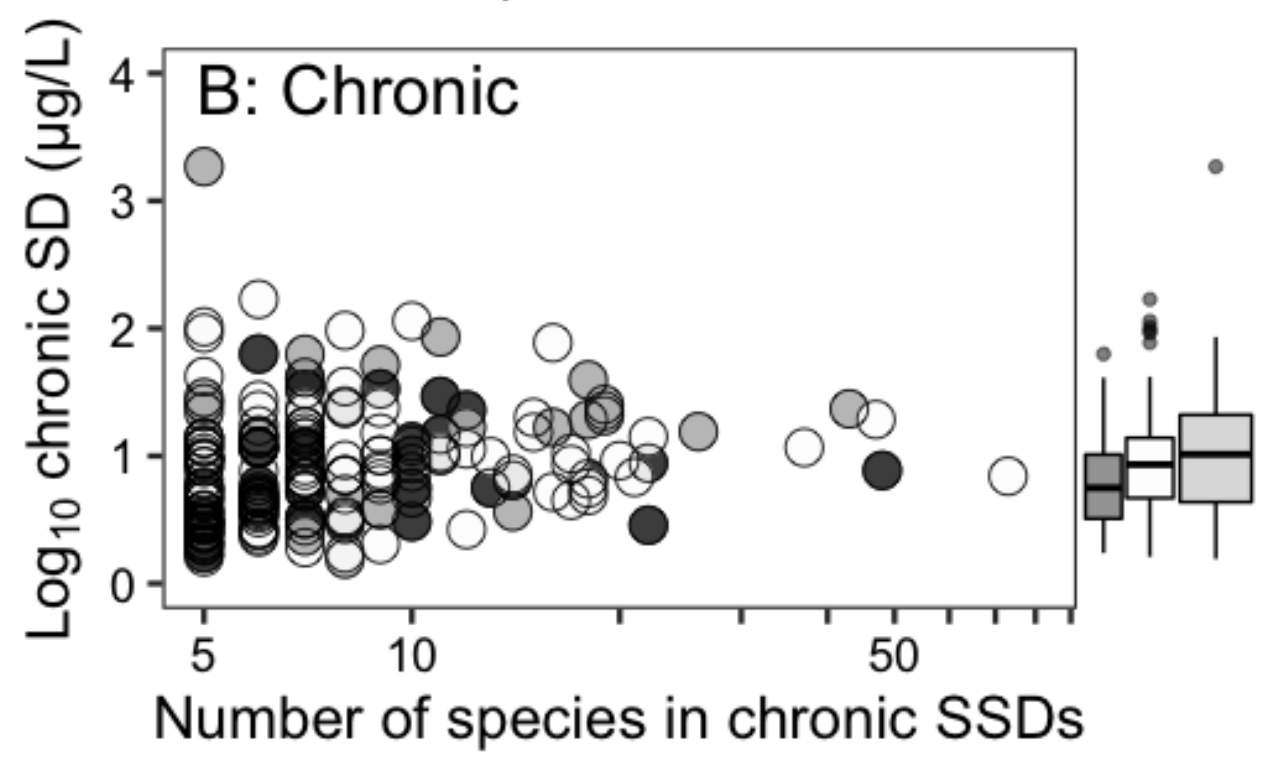

Figure S6. Relationships between the standard deviations (SDs) of (A) 831 acute and (B) 164 chronic species sensitivity distributions (SSDs) and the number of test species in each SSD. White, gray, and black represent unclassified, specifically acting, and narcotic chemicals, respectively. Box plots show the distribution of SDs for the three modes of action. 


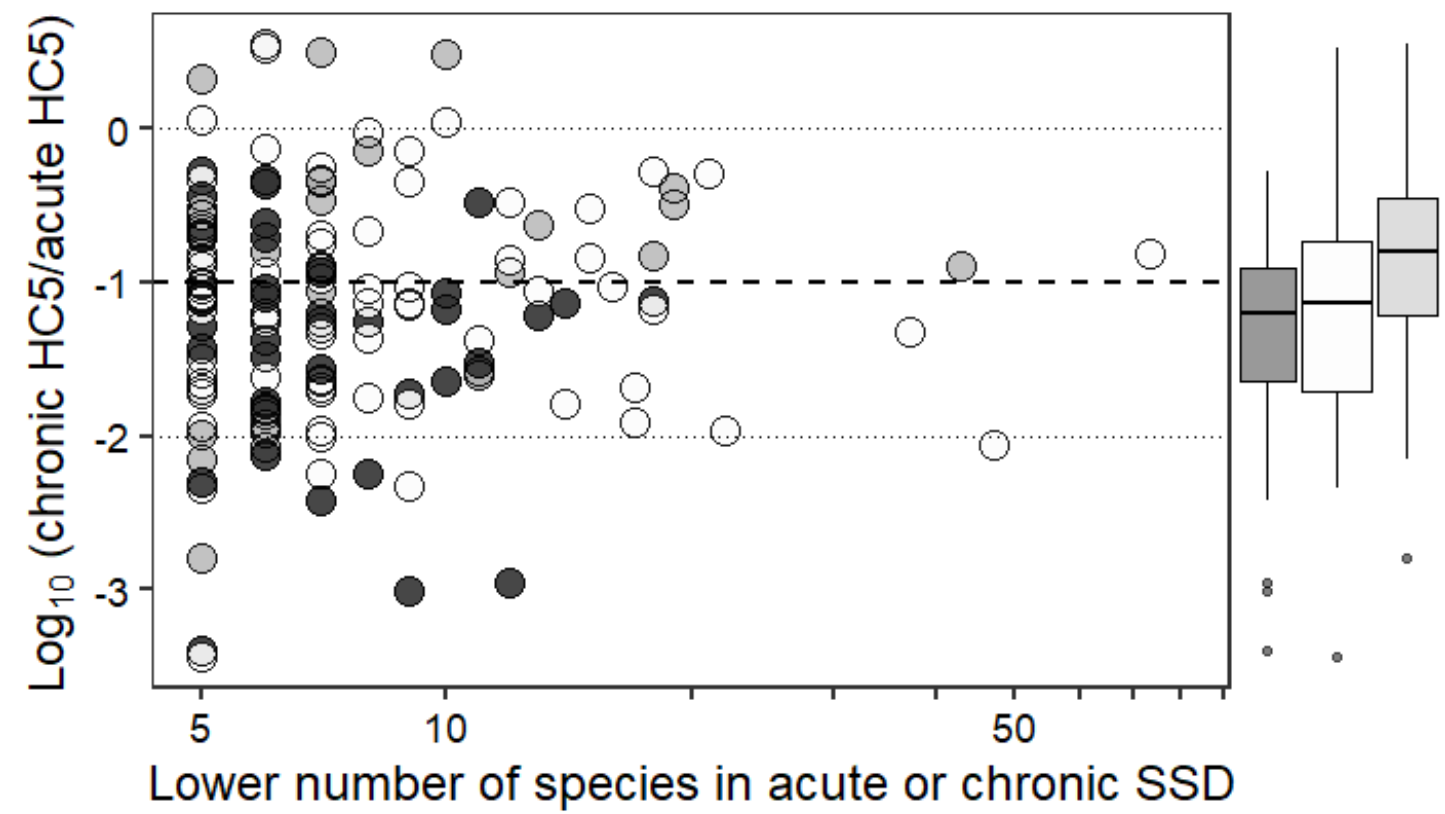

Figure S7. Relationship between the lower number of species in acute or chronic species sensitivity distributions (SSDs) and the chronic to acute hazardous concentration $5 \%$ of the species (HC5) for 150 chemicals. White, gray, and black represent unclassified, specifically acting, and narcotic chemicals, respectively. A dashed line indicates a ratio of 0.1 , and dotted lines indicate ratios of 1 and 0.01 . Box plots show the distribution of differences in HC5 between acute and chronic SSDs for the three different modes of action. 


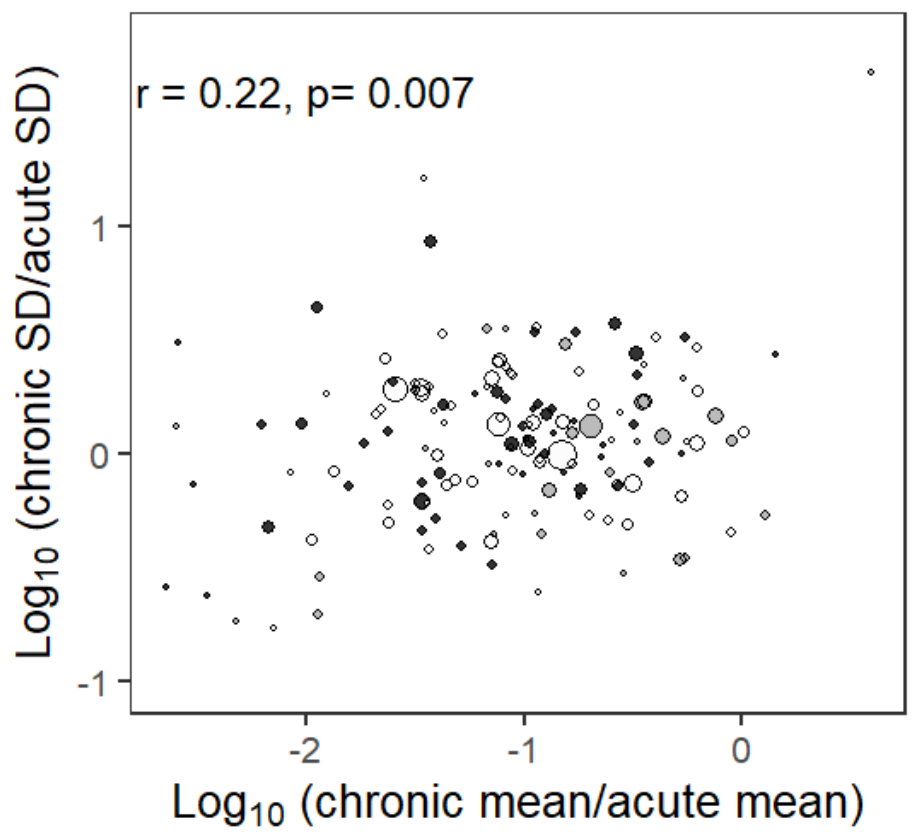

Figure S8. Relationship between the ratios of chronic to acute species sensitivity distribution (SSD) means and the ratios of chronic to acute SSD standard deviations (SDs) for 150 chemicals. White, gray, and black dots (and box plots) represent unclassified, specifically acting, and narcotic chemicals, respectively. Dot sizes represent the number of species in acute or chronic SSDs, whichever is lower. 


\section{An example of $R$ code}

\#\#\# 1: Install and load the necessary packages \#\#\#

install.packages("openxlsx")

install.packages("tidyverse")

install.packages("ggplot2")

install.packages("smatr")

install.packages("EnvStats")

install.packages("ggExtra")

library(openxlsx)

library(tidyverse)

library(ggplot2)

library(ggExtra)

library(smatr)

library(EnvStats)

\#\#\# 2: Import the example dataset \#\#\#

\# This dataset "example.xlsx" includes 20,000 test records randomly selected from the "EnviroTox" database only for demonstration.

\# All the data used in this study was collected from the "EnviroTox" database and please contact the authors if you like to exactly reproduce our results.

EnviroTox_test <- read.xlsx("example.xlsx", sheet=1)

EnviroTox_chem <- read.xlsx("example.xlsx", sheet=2)

EnviroTox_taxo <-read.xlsx("example.xlsx", sheet=3)

\#\#\# 3: Select and process the data \#\#\#

EnviroTox_test_selected <- EnviroTox_test \%>\%

filter (Test.statistic=="EC50" \& Test.type=="A" | Test.statistic=="LC50" \&

Test.type=="A" | Test.statistic=="NOEC" \& Test.type=="C" |

Test.statistic=="NOEL" \& Test.type=="C") \%>\%

filter (Effect.is.5X.above.water.solubility $==" 0 ") \%>\%$

mutate (original.CAS $=$ EnviroTox_chem[match.$\$ C A S$,

EnviroTox_chem\$CAS),"original.CAS"] ) \%>\% 
mutate_at(vars(Effect.value), as.numeric) $\%>\%$

mutate $($ Effect.value $=$ replace $(\$ E f f e c t . v a l u e$, !is.na(.\$Effect.value), .\$Effect.value $*$ $\left.\left.10^{\wedge} 3\right)\right) \%>\% \quad \#$ transform unit $(\mathrm{mg} / \mathrm{L}$ to $\mathrm{ug} / \mathrm{L})$

mutate $($ Unit $=$ replace $($ Unit, Unit=="mg/L", $\mu \mathrm{g} / \mathrm{L} "))$

\# calculate geometric mean and select chemicals analyzed based on the number of species EnviroTox_test_selected 2 <- aggregate(EnviroTox_test_selected\$Effect.value,

by=list(original.CAS = EnviroTox_test_selected\$original.CAS,

Test.type=EnviroTox_test_selected $\$$ Test.type,

Latin.name=EnviroTox_test_selected\$Latin.name), function(x) geoMean $(\mathrm{x})) \%>\%$

rename $($ Effect.value $=\mathrm{x}) \%>\%$

mutate (Trophic.Level = EnviroTox_taxo[match (.\$Latin.name,

EnviroTox_taxo\$Latin.name),"Trophic.Level"] ) \%>\%

group_by(original.CAS,Test.type) $\%>\%$

filter $(\mathrm{n}()>=5)$

\# goodness-of-fit test

EnviroTox_test_fitness_sw <- aggregate(EnviroTox_test_selected2\$Effect.value,

by=list(original.CAS=EnviroTox_test_selected2\$original.CAS,

Test.type=EnviroTox_test_selected2\$Test.type),

function(x) shapiro.test $(\log 10(\operatorname{as} . n u m e r i c(x))) \$ p . v a l u e \quad) \%>\%$

dplyr::rename(p_value $=x) \%>\%$

mutate(adj_p = p.adjust(p_value,"holm"))

not_fit_chem <- EnviroTox_test_fitness_sw[EnviroTox_test_fitness_sw\$adj_p < 0.05 ,"original.CAS"]

\#\#\# 4: SSD estimation \#\#\#

EnviroTox_ssd <-aggregate(x=as.numeric(EnviroTox_test_selected2\$Effect.value),

by=list(original.CAS=EnviroTox_test_selected2\$original.CAS,

Test.type=EnviroTox_test_selected2\$Test.type),

$\mathrm{FUN}=$ function $(\mathrm{x})$ mean $(\log 10(\mathrm{x}))) \quad \%>\%$

mutate(sd=aggregate(EnviroTox_test_selected2\$Effect.value,

by=list(EnviroTox_test_selected2\$original.CAS,

EnviroTox_test_selected2\$Test.type),

function(x)

$\operatorname{sd}(\log 10(\mathrm{x})))[, 3] \quad) \quad \%>\%$ 


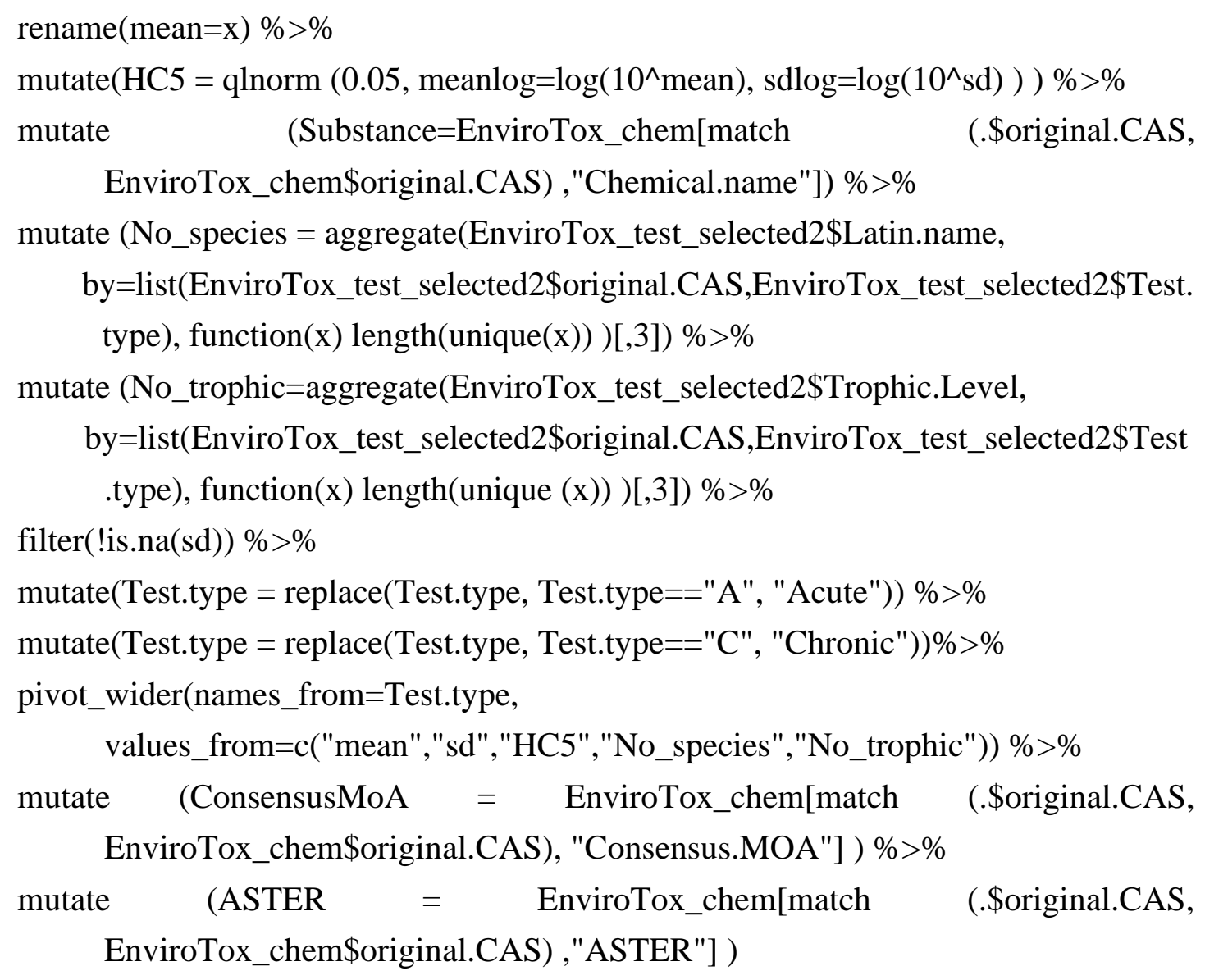

EnviroTox_ssd\$ConsensusMoA <- replace (EnviroTox_ssd\$ConsensusMoA, which(EnviroTox_ssd\$ConsensusMoA=="N"),"Narcotic")

EnviroTox_ssd\$ConsensusMoA <- replace (EnviroTox_ssd\$ConsensusMoA, which(EnviroTox_ssd\$ConsensusMoA=="U"),"Unclassified")

EnviroTox_ssd\$ConsensusMoA <- replace (EnviroTox_ssd\$ConsensusMoA, which(EnviroTox_ssd\$ConsensusMoA=="S"),"Specifically acting")

EnviroTox_ssd\$ConsensusMoA <- as.factor(EnviroTox_ssd\$ConsensusMoA)

\#\#\# 5: Select chemicals analyzed based on results of Shapiro-Wilk test and proportions of taxonomic groups \#\#\#

EnviroTox_ssd_HH <- EnviroTox_ssd \%>\%

filter(! original.CAS \%in $\%$ not_fit_chem ) $\%>\%$

filter (No_trophic_Acute $>=2 \&$ No_trophic_Chronic $>=2$ ) $\%>\%$

separate (Substance, into=c("Short_name"), sep=";", extra="drop") 
\# Check the number of SSDs

\# Acute and chronic SSDs for a total of 33 chemicals were derived in this case.

dim(EnviroTox_ssd_HH)

\#\#\# 6: Fig.1A Relationship between chronic and acute SSD means \#\#\#

\# standardized major axis regression

fit_mean_sma $<-$ sma $($ data=EnviroTox_ssd_HH, mean_Chronic $\sim$ mean_Acute $)$

\# plot

mean_plot <- ggplot(data=EnviroTox_ssd_HH, aes(x=mean_Acute, y=mean_Chronic, size $=$ pmin $($ No_species_Chronic,No_species_Acute) $))+$

geom_point $($ alpha $=0.9$, aes $($ color $=$ ConsensusMoA) $)+$

geom_point(alpha $=0.9, \mathrm{pch}=21)+$

geom_abline $($ slope $=1$, intercept $=0$, size $=0.5,1$ ty $="$ dotted" $)+$

geom_abline $($ slope $=1$, intercept $=-1$, size $=0.5$, tty $=$ "dashed" $)+$

geom_abline $($ slope $=1$, intercept $=-2$, size $=0.5$, lty $="$ dotted" $)+$

theme_bw(base_size $=20)+$

theme $($ axis.text = element_text(color="black"), panel.grid=element_blank( $)$,

legend.position $=$ 'none' $)+$

scale_color_grey $($ start $=0.2$,end $=0.99)+$

xlab(expression(paste( $\log [10], "$ acute mean $(\mu \mathrm{g} / \mathrm{L}) ")))+$

ylab(expression(paste $(\log [10], "$ chronic mean $(\mu \mathrm{g} / \mathrm{L}) ")))+$

geom_abline(intercept=fit_mean_sma\$coef $[[1]][1,1]$,

slope=fit_mean_sma\$coef[[1]][2,1], col="black", size=1.2)

ggMarginal(mean_plot, type $=$ "box", margins $=$ "both", size $=5$, groupFill $=$ TRUE)

\#\#\# 7: Fig. 1B Relationship between chronic and acute SSD SDs \#\#\#

\# standardized major axis regression

fit_sd_sma $<-$ sma(data=EnviroTox_ssd_HH, sd_Chronic $\sim$ sd_Acute)

\#plot

sd_plot <- $\quad$ ggplot(data=EnviroTox_ssd_HH, aes(x=sd_Acute, $\quad \mathrm{y}=$ sd_Chronic, size=pmin(No_species_Chronic, No_species_Acute) ) )+

geom_point(alpha $=0.9$, aes (color=ConsensusMoA) $)+$ 


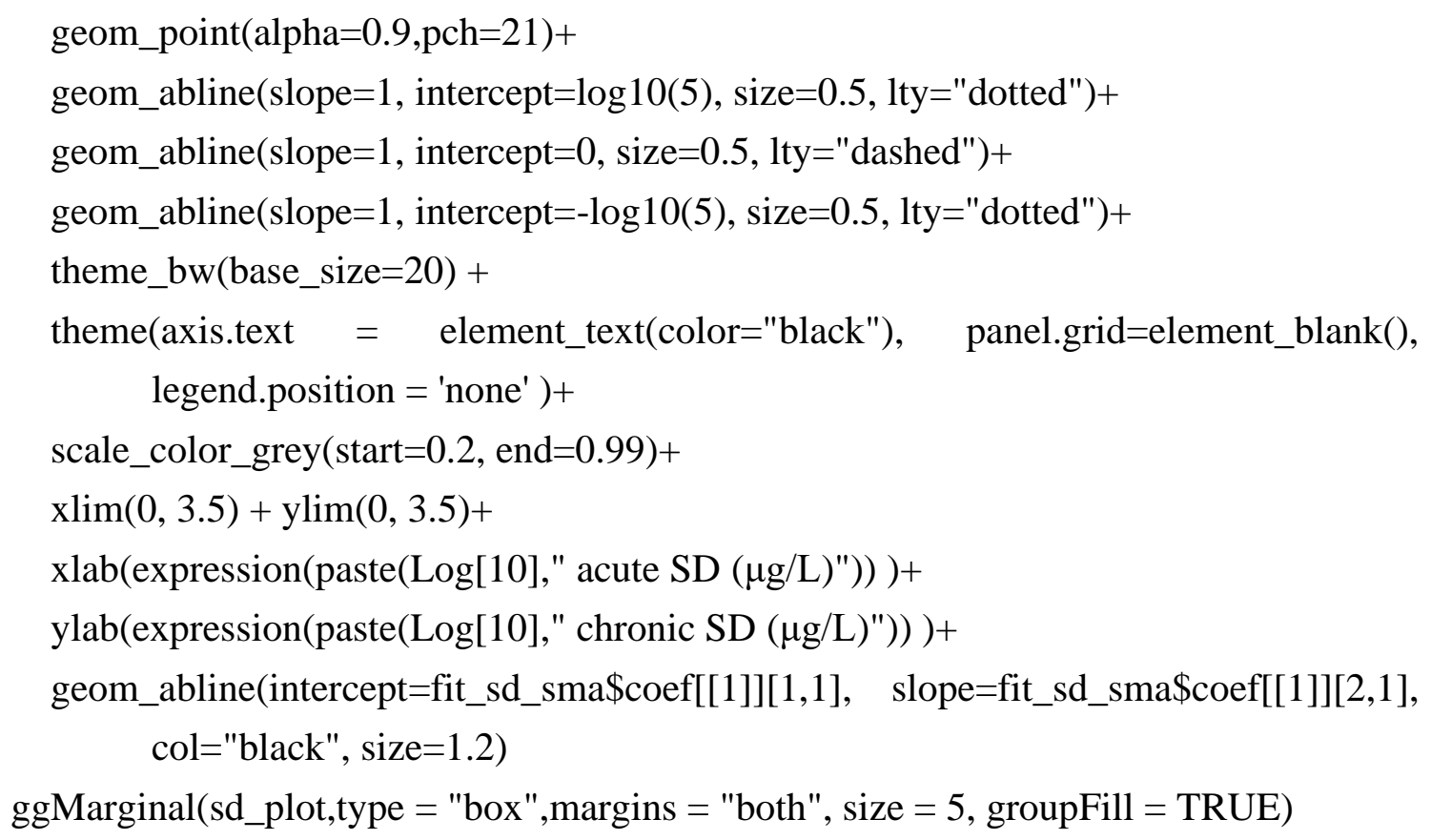

\#\#\# 8: Fig. 2 Relationship between the number of species and ratios of chronic to acute

$$
\text { SSD parameters \#\#\# }
$$

\#Fig. 2A

mean_species <-ggplot(data=EnviroTox_ssd_HH, aes(y=mean_Chronic - mean_Acute, $\mathrm{x}=$ pmin(No_species_Chronic, No_species_Acute) $))+$ geom_point $($ alpha $=0.9, \operatorname{size}=5$, aes $($ color $=$ ConsensusMoA $))+$ geom_point $($ alpha $=0.9$, size $=5, \mathrm{pch}=21)+$ geom_abline $($ intercept $=0$, slope $=0$, lty="dotted" $)+$ geom_abline $($ intercept $=-2$, slope $=0,1$ ty $="$ dotted" $)+$ geom_abline $($ slope $=0$, intercept $=-1$, lty $="$ dashed", size $=0.8)+$ scale_color_grey $($ start $=0.2$,end $=0.99$, name $=" M o A ")+$ scale_x_continuous $($ breaks $=c(5, \operatorname{seq}(10,100$, by $=10))$, labels=c("5","10",rep("",3),"50",rep("",5)), limits=c(5,80),trans="log10")+ theme_bw(base_size $=18)+\operatorname{ylim}(-3,1)+$ theme(axis.text = element_text(color="black"), panel.grid=element_blank(), legend.position="none", axis.title.x=element_blank ()$)+$ xlab("")+ ylab(expression(paste(Log[10]," (chronic mean/acute mean)")) ) ggMarginal(mean_species, type = "box", margins="y", size = 5, groupFill = TRUE) 
\#Fig. 2B

sd_species <- $\quad$ ggplot(data=EnviroTox_ssd_HH, aes(y=sd_Chronic - sd_Acute, $\mathrm{x}=$ pmin(No_species_Chronic, No_species_Acute) ))+ geom_point $($ alpha $=0.9$, size $=5$, aes $($ color $=$ ConsensusMoA $))+$ geom_point $($ alpha $=0.9$, size $=5, \mathrm{pch}=21)+$ geom_abline $($ slope $=0$, intercept $=0,1$ ty $=$ "dashed", size $=0.8)+$ geom_abline(slope $=0$, intercept $=-0.7$, lty="dotted")+ geom_abline (intercept $=0.7$, slope $=0,1$ ty $=$ "dotted")+ scale_color_grey(start $=0.2$, end $=0.99$, name $=" M o A ")+$ scale_x_continuous $($ breaks $=c(5, \operatorname{seq}(10,100$, by $=10))$, labels=c("5","10",rep("",3),"50",rep("",5)), limits=c(5,80),trans="log10")+ theme_bw(base_size $=18)+$ $\operatorname{ylim}(-1,2)+$

theme(axis.text = element_text(color="black"), panel.grid=element_blank(), legend.position="none")+

xlab("Lower number of species in acute or chronic SSDs")+ ylab(expression(paste(Log[10]," (chronic SD/acute SD)")) ) ggMarginal(sd_species, type = "box", margins="y", size = 5, groupFill = TRUE)

\#\#\# 9: Fig.3 Relationship between chronic and acute HC5 \#standardized major axis regression

fit_hc5_sma <- sma (data=EnviroTox_ssd_HH, $\log 10($ HC5_Chronic) $\log 10($ HC5_Acute) )

\#plot

HC5_plot <- $\quad$ ggplot(data=EnviroTox_ssd_HH, aes(x=log10(HC5_Acute), $\mathrm{y}=\log 10($ HC5_Chronic), $\quad$ size=pmin(No_species_Chronic,

No_species_Acute) ) )+

geom_point $($ alpha $=0.9$, aes $($ color $=$ ConsensusMoA $))+$

geom_point(alpha $=0.9$, pch=21)+

geom_abline $($ slope $=1$, intercept $=0$, size $=0.5$, lty $=$ "dotted" $)+$

geom_abline $($ slope $=1$, intercept $=-1$, size $=0.5$, lty $=$ "dashed") +

geom_abline $($ slope $=1$, intercept $=-2$, size $=0.5$, lty="dotted" $)+$

theme_bw(base_size $=20)+$

$x \lim (-4,6)+y \lim (-4,6)+$ 


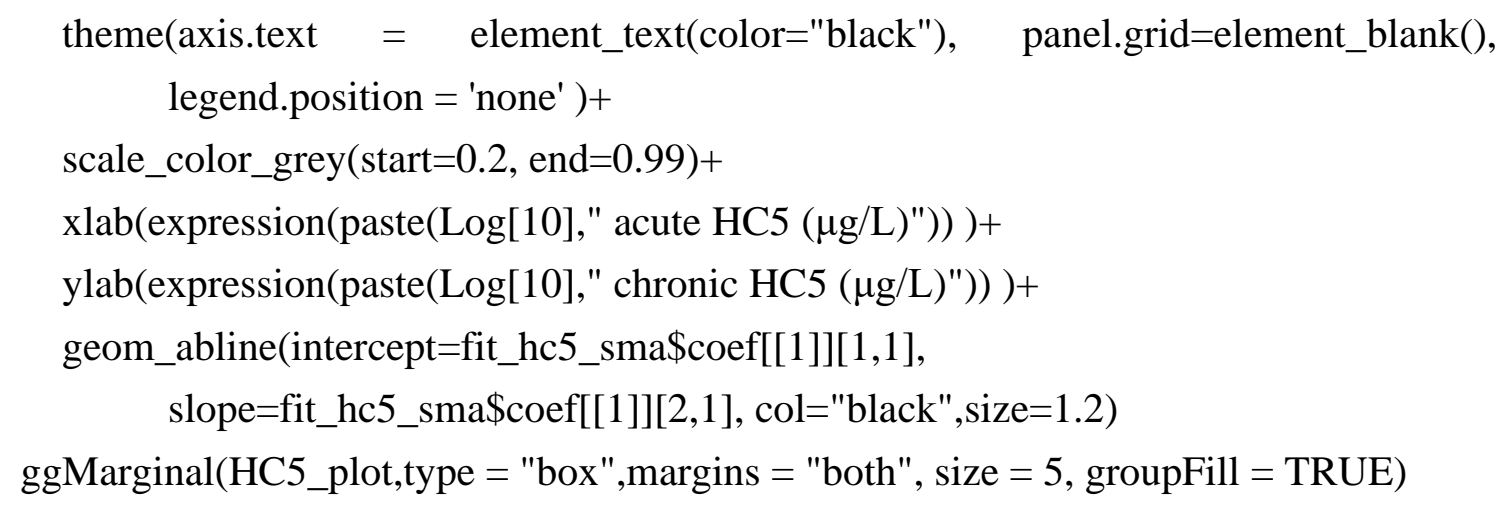

